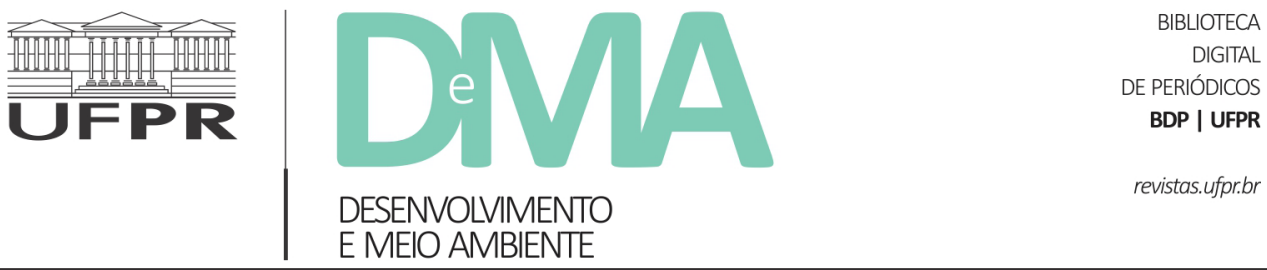

\title{
El (neo)extractivismo y su impacto en la vida de las mujeres en el sudeste de Pará
}

\section{O (neo)extrativismo e seu impacto nas vidas das mulheres no sudeste do Pará}

\section{(Neo)extractivism and its impact on the lives of women in the southeast of Pará State (Brazil)}

\author{
María de los Angeles GUEVARA ${ }^{1 *}$, Edma Silva MOREIRA² \\ ${ }^{1}$ Universidade Federal de Lavras (UFLA), Lavras, MG, Brasil. \\ ${ }^{2}$ Universidade Federal do Sul e Sudeste do Pará (Unifesspa), Marabá, PA, Brasil. \\ *E-mail de contato: ariasguevara2011@gmail.com
}

Artigo recebido em 17 de abril de 2019, versão final aceita em 8 de maio de 2020, publicado em 5 de outubro de 2020.

RESUMEN: Los emprendimientos económicos que a nombre del "desarrollo" se despliegan en el sudeste del Pará desde los años 80 , generan constantes conflictos socioambientales, desterritorializan, y trastocan identidades, dejando sus señales en los cuerpos de hombres y mujeres atrapados por la violencia de un modelo que los usa y los descarta en la misma medida que mercantiliza la naturaleza. Resultante del (neo)extractivismo las mujeres vivencian situaciones que las lleva a problematizar sobre su condición y posición como mujeres. El objetivo propuesto es comprender la experiencia y agencia de las mujeres rurales en áreas impactadas por la minería en el sudeste paraense. La perspectiva seguida se apoya en las Epistemologias del Sur que permiten la construcción de un conocimiento situado que brota del tiempo compartido, de valorización de los saberes anclados en la experiencia de las mujeres, vigilando lo específico que puede informar una lectura interseccional. La metodología emerge de la postura etnográfica seguida que valorizó el contacto directo con la cotidianidad vivida, a partir de las cuales se reflexiona sobre conflictos y resistencias. Se concluye que los grupos de mujeres estudiados en el sudeste paraense, se apoyan en el uso de los bienes que brinda el bioma amazónico y por tanto, están interesados en su gestión sostenible. Muestran el potencial de estrategias apoyadas en la solidaridad, que las lleva a reflexionar como sujetos políticos. La diversidad de trayectorias de vida marcadas por el movimiento espacial, no es obstáculo para articular acciones colectivas, expresión de sus agencias frente al modelo hegemónico capitalista y patriarcal que las oprime. Los grupos estudiados 
constituyen espacios de participación democrática, de solidaridad, autonomía y expresión de la agencia de las mujeres frente a los conflitos socioambientales y la violencia que sufren, reconstruyendo así otros modos de ser y de relacionarse con la naturaleza.

Palabras-clave: neoextractivismo; mujeres rurales; conflictos socioambientales; desarrollo; amazonia oriental.

RESUMO: Os empreendimentos econômicos que, em nome do "desenvolvimento", foram implantados no sudeste do Pará, desde a década de 1980, geram conflitos socioambientais constantes e desterritorializam homens e mulheres, deixando-os presos pela violência de um modelo de desenvolvimento que os usa e os descarta, na mesma medida que mercantiliza a natureza. Como resultado do (neo)extrativismo, as mulheres vivenciam situações que as levam a problematizar seus status e posições como mulheres. O objetivo deste artigo é compreender a experiência e agência das mulheres rurais em áreas atingidas pela mineração no sudeste paraense. A perspectiva adotada baseia-se nas Epistemologias do Sul, que permitem a construção de um saber situado que brota do tempo compartilhado, de valorização do conhecimento ancorado nas experiências das mulheres, monitorando a especificidade que uma leitura intersetorial pode informar. A metodologia emerge da postura etnográfica que valoriza o contato direto com o cotidiano vivido, a partir do qual se reflete os conflitos e as resistências. Concluise que os grupos de mulheres estudados no sudeste paraense dependem do uso dos bens fornecidos pelo bioma amazônico e, portanto, estão interessados em seu manejo sustentável. Eles mostram o potencial das estratégias apoiadas pela solidariedade, o que os leva a refletir como sujeitos políticos. A diversidade de trajetórias de vida marcadas pelo movimento espacial não é obstáculo para articular ações coletivas, expressões de suas agências diante do modelo hegemônico capitalista e patriarcal que os oprime. Os grupos estudados constituem espaços de participação democrática, solidariedade, autonomia e expressão da atuação da mulher diante dos conflitos socioambientais e da violência que lhes acometem, reconstruindo, assim, outras formas de ser e de se relacionar com a natureza.

Palavras-chave: neoextrativismo; mulheres rurais; conflitos socioambientais; desenvolvimento; amazônia oriental.

ABSTRACT: The economic ventures that, in the name of "development," have been implemented in the southeastern of Pará State (Brazil) since the 1980s generate constant social and environmental conflicts and deterritorialize men and women, leaving them trapped by the violence of a development model that uses them and discards them, to the same extent that it commodifies nature. As a result of (neo)extractivism, women experience situations that lead them to question their status and positions as women. This article aims to understand the experience and agency of rural women in areas affected by mining in Southeast Para. The perspective adopted is based on the Epistemologies of the south, which allow the construction of a situated knowledge that sprouts from shared time, valuing knowledge anchored in the women's experiences, monitoring the specificity that an intersectoral reading can inform. The methodology merges from the ethnographic posture that values direct contact with daily life, from which conflicts and resistances are reflected. It is concluded that the groups of women studied in southeastern Pará depend on the use of goods provided by the Amazon biome and, therefore, are interested in its sustainable management. They show the potential of strategies supported by solidarity, which leads them to reflect as political subjects. The diversity of life trajectories marked by the spatial movement is not an obstacle to articulate collective actions, expressions of their agencies in the face of the hegemonic capitalist and patriarchal model that oppresses them. The groups studied constitute spaces for democratic participation, solidarity, autonomy and expression of women's actions in the face of socio-environmental conflicts and violence that affect them. Thus, reconstructing other ways of being and relating to nature.

Keywords: (neo)extractivism; rural women; socio-environmental conflicts; development; eastern amazon. 


\section{Introducción}

Un territorio como el sudeste del Pará ${ }^{1}$ resulta estratégico en la geopolítica mundial, caldo de cultivo para la reconcentración de la tierra en función de intereses globales con la anuencia del Estado y de las élites locales. Estos procesos son operados a la sombra del poder, cuyos ejemplos se encuentran siempre en las narrativas de las mujeres. Las prácticas de la agropecuaria extensiva, el agronegócio, la minería, la explotación forestal, las grandes obras hidroeléctricas y de infraestructura vial tienen consecuencias desvastadoras para la biodiversidad en su totalidad, incluyendo los pueblos tradicionales, indígenas y campesinos; provocando, desplazamientos forzados, destruyendo sus modos y medios de vida rural, contaminando las fuentes de agua y el aire que respiran (Hebette, 2004; Ikeda, 2014; Congilio \& Moreira, 2017; Curvina \& Moreira, 2018).

El (Neo)extractivismo es presentado como una modalidad de la acumulación disfrazada en el discurso sobre el desarrollo; "versión contemporánea del desarrollismo propia de América del Sur, donde se mantiene el mito del progreso y del desarrollo bajo una nueva hibridación cultural y política" Gudynas (2009a). Siguiendo a este autor el nuevo extractivismo fragmenta y desterritorializa, genera un entramado de enclaves y conexiones con los mercados globales que agravan las tensiones territoriales.
Teniendo en cuenta, que la obtención de renta y la solución de muchos de los problemas de salud de las poblaciones amazónicas dependen del conocimiento y gestión del bioma, un conocimiento tradicional, fundamentalmente en poder de las mujeres en su condición de agricultoras, parteras, curanderas, monjas, entre otras, condujo a interrogarnos ¿cómo se relaciona esta realidad con la vida de las mujeres? y ¿por qué los conflictos socioambientales ${ }^{2}$ que genera la mineria son también una cuestión feminista? lo que nos permitió analizar cómo las desigualdades se materializan en sus cuerpos. Una realidad en la que se interrelacionan varias formas de poder jerárquico entre el colonialismo, capitalismo y el heteropatriarcado. De ahí que las experiencias de las mujeres son relevantes para visibilizar su capacidad de agencia frente a los conflitos socioambientales y la violencia que afecta sus vidas.

El contacto directo con las mujeres rurales determinó el rumbo metodológico a seguir. Sus espacios de enunciación fueron la ventana por la que nos asomamos a un mundo más amplio, a través de sus voces era posible reflexionar sobre conflictos y violencias que genera la expansión del capital en el sudeste del Pará. Con ello consideramos este relacionamiento como locus principal en la producción de conocimiento.

\footnotetext{
${ }^{1}$ El sudeste de Pará está conformado por 14 municipios: São Domingos do Araguaia, Bom Jesus do Tocantins, Nova Ipixuna, Canaã dos Carajás, Palestina do Pará, Marabá, São João do Araguaia, Brejo Grande do Araguaia, Curionópolis, São Geraldo do Araguaia, Itupiranga, Eldorado do Carajás, Piçarra y Parauapebas. Luego del lanzamiento del Programa Territórios da Cidadania en el 2008, fueron añadidos Bom Jesus do Tocantins, Brejo Grande do Araguaia, Canaã dos Carajás, Curionópolis, Palestina do Pará, Piçarra y São Geraldo do Araguaia. Este territorio abarca un espacio de aproximadamente $54.469 \mathrm{~km}^{2}$, con una población estimada en más de 617 mil habitantes (IBGE, 2015).

${ }^{2}$ Por conflictos socioambientales asumimos el sentido utilizado por Maristella Svampa (2011) como aquellos ligados al acceso, conservación y control de los recursos naturales, que suponen por parte de los actores enfrentados, intereses y valores divergentes en torno a los mismos, en un contexto de asimetría de poder. Los mismos contienen diferentes lengualjes de valoración respecto al território y al ambiente
} 
El trabajo de campo realizado entre 2016-2017 valorizó el contacto directo con la cotidianidad, el diálogo a través del cual las mujeres construian una narrativa sobre sus trayectorias. Se incluyó además, la observación de sus espacios de vida y de trabajo, y las notas en el diario de campo, a partir de las cuales era posible reflexionar sobre interacciones y conflitos. Resaltamos que, para resguardar el anonimato de los entrevistados, sus identificaciones son colocadas por números.

Todo ello permitió construir un conocimiento que brota del tiempo compartido, de la valorización de saberes anclados en la experiencia, vigilando lo específico que puede informar una lectura interseccional trabajada en sus espacios de vida. Proceso que articula largas conversaciones en que se desencadenan palabras que luego fueron tejidas y encadenadas para que sus voces y sus acciones estuvieran presentes en el texto que se presenta. Nuestra atención fue direcionada a las mujeres organizadas en el "Movimiento das Mulheres Quebradeiras de Coco Babaçu" al Grupo de Economía Solidaria "Bio Saúde-Xarope e infusão de chas" y la cooperativa de mujeres "Sabores da Serra", estos últimos en el municipio Curionópolis, en el sudeste paraense.

El artículo está estructurado en cinco partes incluyendo la introducción. Inicialmente, reflexionamos sobre el (neo)extractivismo y sus efectos en la vida cotidiana de las mujeres, presentando situaciones prácticas, que, muestran su afirmación como sujeitos históricos. Los espacios socioproductivos como resultado de sus acciones colectivas posibilita la construcción de territorios.

En los tópicos siguientes, presentamos, de forma crítica, como las acciones colectivas desarrolladas por esas mujeres, revelan los conflictos entre las lógicas de sustentabilidad y la lógica de la acumulación, en este caso, centrada en la explotación de bienes naturales - el mineral. Así como, revelamos las señales de la violencia, que la sociedad patriarcal y capitalista imprime cotidianamente en cuerpos que encontram fuerza para sobrevivir en un contexto de profundo desiquilibrio económico-social, cultural y ambiental.

Concluimos que sus narrativas nos permiten comprender la experiencia y agencia de mujeres cuya estrategia colectiva expresa resistencia a la rapacidad con que el modelo dominante mercantiliza la naturaleza y la salud humana, asumiendo que lo natural también es político. Las maneras de gestionar, producir, de compartir e intercambiar aspectos materiales y espirituales constituyen chispazos de solidaridad, una flor nacida en el estercolero neoliberal. Sus prácticas están vinculada al cuidado, a la reproducción social de la vida y a una economía cuya importancia quedó invisibilizada en la reproducción del capital.

\section{Las marcas del (neo)extractivismo en la vida de mujeres rurales del sudeste del Pará: experiencia y agencia}

La Amazonia Oriental, contiene la mayor reserva de hierro del mundo, y otros minerales como oro, manganeso, cobre, níquel, cuya extracción y transformación mineral se efectiva por la Compañía Vale, que desde 1985 comenzó a operar en el Gran Complejo Carajas, lo que demandó una gran infraestructura a su servicio que incluyó la hidroeléctrica de Tucuruí, siderurgias, carbonerías, además de carreteras y de una ferrovía de $892 \mathrm{~km}$ que alcanza el Puerto de Maderas en San Luis en Maranhão, Brasil (Hall, 1991). 
La riqueza que sale de este território puede ser contrastada con la pobreza que queda, si se tiene en cuenta los más de 1,5 millones de personas que en Pará viven en condiciones de miséria, un 18,9\% de la población, a los que se le puede sumar unos 1,7 millones en esas condiciones en el Estado de Maranhão, unos 25,7\% del total de sus habitantes (Pinassi \& Cruz Neto, 2015, p. 86). Sin considerar, que la prospectiva hasta el 2030 con el proyecto S11D, duplicará muchas de las inversiones realizadas, entre ellas, la vía férrea y la extracción de unos 90 millones de toneladas métricas de hierro, hasta alcanzar la capacidad de 230 millones por año (Coelho, 2015, p. 64). Lo que convierte a la Empresa Vale, en la mayor mineradora del mundo, y también en la mayor latifundista del Pará, agudizando los conflictos socioambientales unido a los conflictos por la tierra (Santos, 2018).

Es necesario desmontar la falacia sobre el desarrollo que genera la minería. El proyecto "Gran Carajas" acelera el despojo de pueblos indígenas, de pueblos tradicionales y de familias campesinas. La desterritorialización como acto depredatorio atraviesa los cuerpos y las subjetividades, trastoca identidades, destruye los modos y fuentes de vida, al imponer una ontología que rompe con las maneras históricas en que las comunidades se relacionan con la naturaleza. Se ha comprobado que la ferrovía en su trayecto, afecta la vida de más de un millón de personas en 26 municipios, provoca muertes, y perjudica la sociabilidad de las comunidades (Coelho, 2015, p. 50; Congilio et al., 2019), asimismo, la minería contamina el aire, el agua, propaga enfermedades, mutila y consume vidas, mercantiliza los cuerpos de las mujeres, considerados también como un territorio a ocupar y descartar.
La lógica depredadora de la territorialización del capital acciona variables de una ontología eurocéntrica en la que lo humano y no humano son considerados objeto de dominación, se sacrifican los mundos de vida tradicionales en el relacionamiento con la naturaleza y la naturaleza misma es sólo vista como espacio mercantilizado. Baste citar:

[...] que después de cuatro décadas de explotación en la región de Carajás, el resultado del "progreso" realizado hasta aquí no podría ser peor: más de un millón de hectáreas de castañales destruídos, toda la madera de interés comercial retirada del local, bosques quemados para siembra de pastos, rios y nacientes contaminados. Centenas de produtores rurales, indígenas, representantes de movimientos y de la iglesia amenazados y asesinados [...] (Pinassi \& Cruz Neto, 2015, p. 93).

Sólo en 2016, según apunta la documentación realizada por la Comisión Pastoral de la Tierra el proyecto S11D de la mineradora Vale fue responsable por conflictos que afectaron a 484 familias acampadadas en tierras que disputa esa empresa en los municipios Canaã de Carajas y Paraupebas del sudeste del Pará; asimismo, la Ferrovía Carajas afectó el territorio indígena Mâe Maria de los indios Gaviôes en el municipio Bom Jesus provocando la resistencia de más de 300 familias indígenas. Conflictos por el agua y el territorio envuelven a la Empresa Vale con los indios Xikrin de las aldeas de Cateté y Djudjê-ko en el municipio Paraupebas (CPT, 2017).

Podríamos preguntarnos entonces, a quién desarrolla el "desarrollo" de los megaproyectos mineros, qué desarrollo es ese que desterritorializa, despoja, recoloniza los cuerpos de hombres y mujeres, que priva de los medios de vida y de la vida 
misma, que desestabiliza los ciclos naturales. Es en ese sentido que una lideresa del movimento de expropiados por la hidrelétrica de Tucuruí en Pará, teniendo su territorio totalmente inundado, levanta el cuestionamento sobre el desarrollo que los megaproyectos dicen promover, ella relata:

Yo ni sé si fue desarrollo [...] Es tan dificil para la gente hasta imaginar, será que fue el desarrollo? Porque la gente ve tanto sufrimiento, mismo con ese desarrollo que ellos tienen, tanta gente sufriendo; mucha dificuldad, mucha lucha. Yo se que nuestra vida, fue una vida muy sufrida con ese cambio de entonces para acá. (Entrevistada 1, 74 anos, sudeste de Pará, 11/2017).

Esa lideresa expropriada reconoce que el desarrollo promovido por el emprendimiento hidroeléctrico significó el sufrimiento, por la pérdida de su lugar, de su casa y de su modo de vida, aspectos esos que guardan sentidos y sentimentos profundos como traumas que marcam la vida, los cuerpos y las acciones de quien pierde su lugar, su pertenecimiento y su historia (Curvina \& Moreira, 2018).

La concepción sobre el desarrollo que acompaña a la modernidad occidental ha sido cuestionada por pensadores de diversas áreas del conocimiento que destacan la necesidad de una conceptualización con menos imprecisiones, favoreciendo, así, el encuentro de caminos alternativos para los modelos de sociedad construídos hasta el momento. A partir de los años 70, los cuestionamentos aseveran que el crecimiento industrial y técnico se demuestra inoperante para las sociedades humanas, dada el acelerado proceso de destrucción de la naturaleza, portanto de degradación de las vidas (Peña-Vega, 1997).
El término desarrollo tiene mayor fuerza y contenido al apuntar caminos sustentables que aseguren la superación de las crisis económicas con seguridad y certeza. Sin embargo, son muchas las controversias sobre un camino de desarrollo con una inversión destructiva en las sociedades y la naturaleza, ya que el desarrollo pensado como progreso ilimitado, con sus fórmulas, conceptos y prácticas sociales, políticas y ambientales comenzó a componer un escenario conceptual heterogéneo involucrado en una perspectiva de seguridad para la vida en la sociedad industrial (UNESCO, 1982). Para algunos especialistas esas prácticas se convertían en un mito, o sea, en una perspectiva simplista sobre los rumbos a ser adoptados para una vida sustentable en el planeta. Afirman que ella reduce la naturaleza a una dimensión utilitarista que descarta los saberes tradicionales frente al avance tecnológico e industrial, portanto no consigue avanzar en alternativas más allá del crecimiento económico (Brito, 1999).

En general esa perspectiva simplista tiene un lugar destacado hasta hoy, aún se muestra carente de contenido y prácticas puesto que la racionalidad encantada con la ciencia y la técnica al instrumentalizar lo humano y la naturaleza avanza en la destrucción evocando una estabilidad segura como garantia de un desarrollo sostenible.

En este sentido, el (neo)extractivismo es presentado en el discurso desarrollista como prometedor del crecimiento económico, de empleos y bienestar social escondiendo la otra cara de la ontologia moderna, que "refuerza la idea de manejar a la Naturaleza como una canasta de recursos comercializables que se administra bajo criterios económicos" (Gudynas, 2010, p. 66).

Otras propuestas nos llegan desde una epistemología descolonizadora, que sustenta una recon- 
ceptualización e indica la emergencia de alternativas ante la agonía del mito del desarrollo. Autores como Esteva (2009), Gudynas (2009b), Escobar (2010) expresan un pensamiento latinoamericano denominado "posdesarrollo". ¿Qué grados de veracidad, qué silencios trajo consigo el lenguaje del desarrollo?" Pregunta que nos ayudan a entender una realidad que contradice el supuesto bienestar que ese "desarrollo" traería consigo, según prometia el discurso neodesarrollista.

El feminismo ${ }^{3}$ como corriente de pensamento cuestionó las estrategias de desarrollo seguidas como excluyente a las mujeres, al colocarlas como receptoras pasivas de políticas, subvalorando sus aportes a la riqueza socialmente creada y al mantenimiento social. A partir de los años ochenta posturas posestructuralistas problematizan las desigualdades desde la interseccionalidad de género, raza y clase relacionadas con una crítica al desarrollo. Las críticas más profundas vendrían luego desde la economia feminista, de los feminismos poscoloniales y del ecofeminismo; estos últimos analizan al capitalismo globalizado, el patriarcado y el colonialismo como parte de un mismo sistema de dominación sobre las mujeres, descubriendo a su vez los paralelos históricos, culturales y simbólicos entre la opresión y explotación de las mujeres y de la naturaleza, paralelos que reproducen dicotomias, subordinaciones y jerarquias.

Este tipo de desarrollo ha sido caracterizado como um mal desarrollo, un desarrollo mal orientado, paradigma industrial occidental, no sostenible, cuyas tendencias se orientan hacia un apartheid ambiental (Shiva, 2001).
Una economía basada en valores del patriarcado y del mercado capitalista con énfasis en el crecimiento, como fue dicho arriba, omite y destruye las fuentes de vida de las poblaciones tradicionales en el sudeste del Pará, como por ejemplo, las mujeres que reproducen su vida quebrando el Coco Babaçu, en tanto, "pasa por alto el valor económico de las economías vitales que son necesarias para la supervivencia humana y ecológica: la economía de la naturaleza y la economía del sustento" (Shiva \& Mies, 2013, p. 19).

La modernización del territorio asociado a la presencia de las trasnacionales mineras con anuencia del Estado, deja para las comunidades sus propias marcas: el polvo de hierro que es lanzado al aire todos los dias doce veces con el paso del tren, el agua que es contaminada, el rio que se seca, la carne rasgada, la casa rajada, la herida en la tierra, la biodiversidade desaparecida, la población desplazada, la violencia que mata. "La linea pasa por dentro de nuestro barrio, cortando todo y acabando con todo. Los niños van para la escuela sin seguridad ninguna, con ese gigante. Ya hubo varios accidentes. Nuestras casas, están todas todas quebradas" (Entrevistada 1, Vecina de Marabá, sudeste de Pará, 09/2016). Todo, cuanto aparece disimuladamente escondido en el discurso sobre el desarrollo.

El preguntarnos ¿cómo se relaciona el modelo (neo)extrativista con la vida de las mujeres? nos conduce al análisis del cómo las desigualdades, los conflictos e a violencia se materializan en sus cuerpos. Una realidad que interrelaciona varias formas de dominación, genera experiencias específicas en los diversos grupos de mujeres; lo

\footnotetext{
${ }^{3}$ Estamos considerando al feminismo como una corriente de pensamento extremamente heterogénea, que abriga uma diversidade de posturas, asimismo, el ecofeminismo es diverso, nos atenemos aquí a posiciones del ecofeminismo crítico y tercermundista.
} 
que exige de una mirada interseccional ${ }^{4}$ que de cuenta de las multiples opresiones marcadas por la posición de clase, género, color de la piel, la edad, la sexualidade, entre otras, y a su vez reforzadas por la violencia del poder patriarcal global en los territorios donde la expansión minera suplantó actividades económicas tradicionales, desplazando a las mujeres de aquellos espacios generadores de renta, como puede ser la actividad extractivista de plantas medicinales, del coco babaçu, las frutas o la producción de alimentos, sobredimencionando su lugar en la reproducción de la vida.

En las narrativas siempre es posible encontrar referencia a la dura realidad vivida por las mujeres en la época del garimpo de Serra Pelada 5 . Ellas llegaban de todas partes (en especial del estado de Maranhão) porque sus maridos trabajaban en el garimpo, espacio del actual municipio Curionópolis ${ }^{6}$, cuya economía desde finales de la década de los 70 , durante todo los ochenta y hasta su cierre en 1991 giraba en torno a la explotación minera informal de oro. Otras, eran madres solteras, buscaban alternativas de vida, hacían todo tipo de trabajo, prestaban servicios domésticos, garimpeaban alrededor de la ciudad, eran vendedoras de alimentos o simplemente ejercían la prostitución, pues la explotación sexual de los cuerpos de las mujeres se convirtió en una de las principales fuentes de renta. Una de mis entrevistadas afirmo que: "Durante la época de auge del garimpo, habia en Curionópolis 40 casas de prostitución y ninguna escuela" (Entrevistada 2, 62 años, Curionópolis, 10/2016). La precariedad de las condiciones de vida es narrada por todas las entrevistas realizadas.

Fue una experiencia de mucho sufrimiento. Por la
tarde me iba con las mujeres a garimpear oro y a
la vez vendia bolos y geladinho. Garimpeabamos
y vendíamos. Si los garimperos no tenían dinero

\begin{abstract}
${ }^{4}$ Entre las autoras más significativas en el uso del término está Crenshaw (1991) en el texto "Cartografiando los márgenes. Interseccionalidad, políticas identitarias y violencia contra las mujeres de color". Otro texto útil para el análisis es el de Mohanty (2008) "Bajo los ojos de Occidente Academia Feminista y discurso colonial" en tanto realiza una crítica al carácter homogeneo con que el feminismo occidental trata a las "mujeres del tercer mundo" sin importar la clase social, la ubicación o las contradicciones raciales o étnicas. También resulta de interés el tratamento que al término da Lugones (2008) entendido aquellas "marcas potentes de sujeción o dominación" (raza, género, clase, sexualidad) que actúan de tal forma que ninguna de ellas, al estar oprimiendo, moldea y reduce a una persona sin estar tocada por o separada de las otras marcas, a la que añade las violencias que se infieren desde la dominación global del poder masculino y que resulta las muertes de mujeres en México, Brasil, Sudafrica y en muchas otras partes.

${ }^{5}$ Su inicio fue a finales década del 70, concentró a unos 50, 000 “garimpeiros" llegados de todo el país y procedentes de toda la estructura social. En mayo de 1980 el gobierno realizó intervención, prohibiendo la salida del oro, estabelecendo la exigencia da cartera de garimpeiro y abriendo una agencia de la Caja Económica Federal que realizaba su compra. El garimpo fue explotado manualmente. En cinco años, una sierra de 100 metros de altura fue transformado en un crater de 300 metros de largo por 100 de profundidad, con 1,5 millones de metros cúbicos de agua. Ese material de unos $35 \mathrm{~kg}$ era cargado en las espaldas de los lamados "hormigas" envueltos en lama por escaleras que denominaban "adeus mamãe", por que causaban constantes acidentes y muertes, que no constan en registros oficiales.El mercúrio usado para separar los residuos de oro quedó depositado en rios y lagunas, donde transformado en metil-mercúrio causa hasta la actualidad multiples enfermedades. Con la apertura del garimpo a la entrada de las mujeres en 1986 daría origen a una comunidad donde habitan unas 6000,00 familias, marcada por los conflitos sociales y ambientales, por la pobreza, y la marginalidad, con indicadores epidemiológicos de enfermedades negligenciadas como hansinease (lepra), tuberculosis, sífilis, entre otras. Ver a respecto de Hansiniase a Silvestre \& Lima (2016). El 80\% delas famílias depende hoy del subsidio estatal "bolsa de familia".
\end{abstract}

${ }^{6}$ Este município debe su origen al Garimpo de Serra Pelada, en el Km 30 de la Carretera PA-150 y su nombre al famoso Major Sebastião Curió, repressor da Guerrilla del Araguaia, enviado por el ejército brasileño a la región para contener la tensión social que provocaban los conflitos por la tierra y tomar control de aquel espacio. Fue el primer perfecto de ese município (Kotscho, 1984). 
trocaban bateas de tierra por bolo y geladinho. La gente comenzó a trocar para lavar la tierra. Con ello las mujeres tiraban el sustento de sus hijos, eran pequenas cantidades que daban para el sustento (Entrevistada 3, 47 años, Cuirionópolis, 12/2016).

Otra de mis entrevistadas describe el contexto local en la década de los ochenta como "un espacio dantesco", como un "no lugar", que concentraba personas carentes, sin saneamiento y con una situación educativa "gritante" (Entrevistada 2, 62 años, Curionopolis, 10/2016). Las monjas (freiras) recién llegadas desde el sur del país se encargaron de organizar las primeras escuelas y de habilitar las personas para ejercer el magisterio. "Éramos tres monjas con formación superior, en vez de dedicarnos al trabajo pastoral nos dedicamos a la educación, había mucha hambre, recuerdo que alumnos y maestras desmayaban en la escuela [...] luego de mucho batallar conseguimos la merienda escolar" (Entrevistada 2, 62 años, Curinópolis, 10/2016).

En esas condiciones de exclusión social, de pobreza, de violencia social, y de carencia de espacios educativos, comenzó a gestarse la organización solidaria en lo que hoy se conoce como Curionópolis. Partió de la reunión de las mujeres para discutir su realidad, las enfermedades por la falta de alimentos, de higiene y de agua potable.

Mujeres que llegaron a Serra Pelada, luego de 1986, en que se autorizó su entrada en el área de explotación mineral, describen la complejidad de entrar en un espacio de hombres, más, ellas encontraban allí una oportunidad para la generación de renta, "mientras los hombres en su mayoría traba- jaban en la cava, las mujeres vendían mercadurias traídas de otras partes, lavaban tierra, cocinaban, garimpeaban, otras hacian de prostitutas, mujer de la vida" dice una mujer de 41 años, madre soltera, llegada de Ceará (Entrevistada 4, Serra Pelada, 11/ 2016). Entre ellas, aún es posible encontrar algunas que se dicen dueñas de barrancos ${ }^{7}$ como es el caso de Entrevistada 5, de 63 años, llegada desde Rio Grande do Sul en 1986. “cuando llegué ya mi marido habia muerto en el garimpo. Tengo a mi nombre diez barrancos de cuatro metros cuadrados, soy socia de una cooperativa de garimperos y aguardo por una recompensa que me permita retornar y comprar un lote" (Entrevistada 5, Serra Pelada, 11/ 2016). Otra mujer llegada de Açailandia, estado de Maranhão, cuando contaba con 15 años relata sobre sus miedos:

...cuando llegue aqui, quede impressionada con todo lo que estaba viendo, la cava aún funcionaba, llegue a ver el hormiguero humano, subiendo y descendiendo, todos llenos de lama, llegué aún a descender a la cava [...] tenía mucho miedo, era solo hombres, pocas mujeres, que hacian de todo para sobrevivir (Entrevistada 6, 49 años, Serra Pelada, 02/2017).

Luego del cierre del garimpo en 1991 se agudizaron los conflitos, entre las cooperativas de garimperos, que, entre tanto, se habían organizando detrás del sueño de una recompensa financiera y las empresas trasnacionales, entre ellas, la Vale S.A., a quien el Estado otorgó el derecho de labra. Sierra Pelada, se convierte así en una comunidad rehen de la minería, cuya perspectiva mayor es trabajar con la mencionada empresa, que absorbe y coopta

\footnotetext{
${ }^{7}$ Espacios en que se divide la propiedad en el garimpo para la explotación artesanal del mineral.
} 
a unos pocos, fundamentalmente jóvenes, mientras la mayoría busca sus subsistencia como puede, en el garimpeo informal, y en otras atividades ilegales incluidos el mundo de la droga o la prostitución; los servicios públicos existentes no satisfacen la demanda de empleo, sin que vislumbre el diseño de otro modelo económico. En tales circunstancias, la solidaridad entre las mujeres ha sido fundamental en la búsqueda de formas de sobrevivencia que se expresa en la actividad garimpera informal y en la organización de formas cooperadas de producir.

Mas reciente en el tiempo, en otros municipios del sudeste de Pará como Canaã de Carajas donde la Vale S. A. empreende el mayor projeto minero $\mathrm{S} 11 \mathrm{D}$, las mujeres denuncian los impactos causados por la mineria en la producción rural. La Vale realiza compra ilegal de tierra y despeja familias acampadas o asentadas. Testimonios recogidos por Brito (2016) en estudios realizados por el Istituto Brasilero de Análisis Sociales y Económicos (IBASE), muestran los impactos socioambientales que dicha empresa trajo consigo, además de un desmedido aumento de la poblacional flotante, fundamentalmente de hombres en las primeras fases del proyecto, con lo que se agrega elevados índices de violencia sexual y la aparición de los llamados "filhos da mineração". Otro estudio del mismo instituto muestra las paradojas entre el crecimiento expresivo de las variables económicas, las variables de desarrollo social y de sustentabilidad internacionalmente reconocidas (IBASE, 2018).

El perfil antropocéntrico del arrogante mandato judeo-cristiano no sólo está dirigido a dominar la naturaleza, sino también a las mujeres, consideradas seres humanos "mas débiles o menos inclinados a involucrarse en juegos de poder y domínio" (Max-Neef, 1986, p. 43).

La herida abierta en la amazonia agudiza los conflictos socioambientales, entendidos como aquellas disputas que surgen en torno al uso, acceso y apropiación de los recursos naturales por parte de actores con diferentes niveles de poder e intereses (De la Cuadra, 2015). Ellos resultan de diferentes lógicas ontológicas y epistemológicas que se confrontan en practicas, valores y sentidos otorgados al território, a la naturaleza y a los modos con que nos relacionamos con ella, a cómo comprendemos qué es el progreso, qué es el desarrollo.

La apropiación de los bienes de la naturaleza son cada vez mayores y marcarán el futuro de toda la amazonia y por lo tanto, de los pueblos que historicamente hicieron de ella su casa común. Se opera así, un verdadero proceso de recolonización que desposesiona, desplaza, despoja y desterritorializa, dejando una huella de violencia y de muerte ${ }^{8}$. Es en este contexto que ubicamos la agencia de las mujeres que construyen otras formas de gestionar los bienes de la naturaliza y promover un otro desarrollo.

Desde el punto de vista epistémico, reflexionar sobre este tema trajo a colación la comprensión conceptual realizada por los Feminismos del Sur sobre la agencia, entendida en occidente como sinónimo de resistencia, de autonomía y subversión frente a las relaciones de dominación, desde la influencia ejercida por autores como Foucault. Tanto Mah-

\footnotetext{
${ }^{8} \mathrm{Si}$ queremos dar una mirada a las estadísticas sobre los conflictos en el campo una fuente imprescindible son los informes anuales realizados por la Comisión Pastoral de la Tierra. Del informe de 2017 es posible inferir que el Sudeste del Pará acumuló el 67,6\% de los conflictos por la tierra y por el agua ocurridos en todo el estado
} 
mood (2006) como Mohanti (2008) realizan una crítica al entendimiento del feminismo occidental sobre la comprensión de las mujeres del tercer mundo como "otras" homogéneas, necesitadas de ser liberadas. En el caso de la segunda autora, quien estudió otras modalidades de agencia que se configuran en tradiciones no liberales como el movimiento femenino de las mezquitas en Egipto comprende la agencia como "capacidad para la acción creada y propiciada por relaciones concretas de subordinación históricamente configuradas" (Mahmood, 2006, p. 123), trata así, de superar los binarismos propios del feminismo occidental, aun cuando reconoce y se ve influenciada por los importantes aportes realizados por autoras como Judith Butler. A partir del entendimiento sobre el poder reconceptualiza la agencia "no sólo como un sinónimo de resistencia a las relaciones de dominación, más también como una capacidad para la acción propiciada por relaciones de subordinación específicas" (Mahmood, 2006, p. 133).

En este artículo asumimos la comprensión de agencia que emerge del feminismo decolonial, analizada más en términos de intersubjetividad e interdependencia, permitiendo entender las variaciones en las posicionalidades mediadas por la intercepcionalidad, que imprimen factores como lo clasista, el género, lo racial, lo étnico, lo generacional. La agencia es entendida como capacidad para la acción, para la movilización y la construcción de organizaciones socioproductiva. Por otra parte, el concepto de experiencia ha sido central para el feminismo al dar significación política a lo personal, a la cotidianeidad específica de las relaciones sociales que marcan la vida vivida por las mujeres en lo individual y lo colectivo.

Siguiendo a Teresa de Lauretis la experiencia "es el proceso por el cual la subjetividade es constituída [...] A través de ese proceso una persona se coloca o es colocada en la realidad social" (Lauretis, 1984, p.159). Si consideramos la diferencia como marca que imprime la vida cotidiana como mujeres en contextos de extrema vulnerabilidad, entonces siguiendo a Avtar Brah la experiencia es el proceso de significación, una práctica de dar sentido, tanto simbólica como narrativamente. "la experiencia no refleja una «realidad» ya dada, sino el efecto discursivo de los procesos que construyen lo que llamamos realidad" (Brah, 2011, p. 34).

La búsqueda de la agencia de las mujeres rurales, construida en el marco de las tensiones que generan los conflictos socioambientales, la lucha por el territorio, contra la desteritorialización y la violencia requieren de empatía, para comprender en la opacidad de sus experiencias, el cómo se constituyen sujetos colectivos, cuyas acciones son marcas de sus resistencias en un territorio en acelerado proceso de cambios como el sudeste del Pará.

\subsection{Mujeres del Movimento das Quebradeiras de Coco Babaçu}

Podemos ejemplificar los efectos de la minería y a su vez la búsqueda de otra relación con la naturaleza en las mujeres rurales de comunidades tradicionales identificadas como Quebradoras de Coco Babaçu, su territorio simbólico abarca espacios ubicados en el valle del río Tocantins cuya área de babuçuais ${ }^{9}$ comprende unas 290,000 hectáreas.

\footnotetext{
${ }^{9}$ Denominación utilizada por el Movimiento Interestadual de Quebradeiras de Coco Babaçu (MIQCB) para identificar espacios donde trabajan
} 
Hoy la renta que emerge del extractivismo está amenazada debido al arrendamiento de los babuçuais a empresas que extraen el coco entero para fabricar el carbón que alimenta el parque industrial de la ciudad de Marabá, y de otras industrias ubicadas en el estado de Maranhão. Las llamadas Ferro gusas demandan del carbón para derretir el hierro en sus hornos. Teniendo en cuenta los límites que impone la legislación ambiental para dar continuidad a la desvastación de los bosques amazónicos, las empresas negocian con latifundistas para comprar el coco, convirtiendolo en materia prima estratégica para alimentar sus siderurgias; cuestión que tensiona la existencia de una cultura tradicional que organizó formas específicas de relacionarse con la naturaleza, asimismo, afecta la reproducción social, cultural y física de las mujeres que generaciones tras generaciones garantizaron la sostenibilidad de la vida quebrando el coco babaçu.

Las mujeres que por generaciones han alimentado a sus familias de la renta que emerge de la recolección del coco babaçu, son mujeres en condición de pobreza, cuerpos negros en más de un $90 \%$, sub-escolarizadas, pertenecientes a varias generaciones, marcadas por la violencia patriarcal y del colonialismo interno, y por la expansión del capitalismo trasnacional encarnado en el agronegocio y la explotación mineral que les priva de la fuente de sustento familiar: los cocos babaçu (Guevara, 2018).

Para estas mujeres, el acceso a los babuçuais es colocarse más allá de la economia doméstica, es defender un modo equilibrado de ser y existir en un paisaje del que se consideran parte. Su histórica lucha las llevó a organizarse, primero em sindicatos rurales y luego en Movimiento social, conocido como "Movimiento Interestadual das Quebradeiras de Coco Babaçu" (MIQCB). Creado en 1995, luego de un largo proceso de problematización de sus realidades, de organización y articulación de grupos existentes; constituye la expresión política de la organización de mujeres que realizan esta actividad en los estados de Maranhão, Piauí, Tocantins y Pará. Esta organización reivindica políticas públicas relacionadas a su condición y posición de género como mujeres rurales, desde la racialidad de sus cuerpos, entre ellas, la reforma agraria, y la sostenibilidad de los babuçuais como territorios simbólicos, el derecho a accesar libremente, es decir, la democratización en el acceso y uso como um bien común que la naturaleza regaló y que les asegura a ellas y a sus famílias sobrevivencia.

El MIQCB, articula redes de sociabilidad como asociaciones, clubes, comisiones, grupos de mujeres y cooperativas, constituyendo la representatibidad de las quebradoras como sujetos políticos. Su bandera política de lucha en la actualidad es la concresión práctica del proyecto de Ley "Babaçu Livre" ${ }^{\prime 0}$. La concresión de esta ley enfrenta diretamente a la quebradoras con propietarios de tierra que tratan de evadir su cumplimiento y ejercen todo tipo de violencia contra las mujeres. Situación que refuerza su agencia.

realizamos movilizaciones, vamos a la cámara mu-

las mujeres que lo integran como comunidades tradicionais, sin obedecer a términos geográficos

${ }^{10}$ Proyecto de Ley 231/2007. El proyecto de Ley "Babaçu Livre" es de autoria del diputado Domingos Dutra (PT/MA), esta propuesta prohíbe el derrumbe de las palmas en los estados Maranhão, Piauí, Tocantins, Pará, Goiás y Mato Grosso, y crea las reglas para a explotación de la especie (BRASIL, 2007). 
nicipal. Queremos hacer valer esa ley, los dueños de la tierra están envenenando las palmeras, colocando cerca eléctrica para impedirnos entrar en el territorio, están contratando el coco entero para carbón, no quieren donar el coco para la gente, cuando denunciamos somos perseguidas, amenazan de muerte a nuestras compañeras, los cocos están quedando cada vez más lejos y la prefectura brinda poco apoyo (Entrevistada 7, 27 años, São Domingos do Araguaia, 11/2016).

La violenccia contra las mujeres, es también una violencia que responsabiliza al Estado, es una cuestión de Estado (Segato, 2011) cuando no es capaz de hacer cumplir la legislación aprobada, cuando es indiferente ante un reclamo de apoyo, ante las amenazas y muertes de las mujeres.

somos tan discriminadas que cuando juntamos el coco, muchas veces se estraga y no conseguimos apoyo de la prefectura para transportarlo [...] no vamos a pedir comida, pedimos sí, apoyo para producir [...]. Nuestra lucha es por el babaçu libre, es una cuestión de todas nosotras, queremos a las mujeres y a las palmeras vivas (Entrevistada 8, 60 años, São Domingos do Araguaia, 12/2016).

Este discurso, si bien, por una parte, nos habla de negociaciones, de exigencias, de utopías, también expresa las condiciones de vulnerabilidad en que se encuentran las mujeres y las palmeras. Entre las mujeres y entre las mujeres y las palmeras de Coco Babaçu existe una interdependencia y una ecodependencia puesta en juego por los conflictos socioambientales que definen la expansión del capital en el sudeste del Pará. Siguiendo a (Pérez Orozco, 2013) se trata de un conflicto de lógicas: de la lógica de la acumulación y la lógica de la sostenibilidad de la vida. Y es esa segunda lógica la que nutre las experiencias de las mujeres que quiebran el coco Babaçu.

Los babaçuais pueden ser considerados territorios simbolicamente construidos, a partir de prácticas sociales que conforman contextos particulares de relación entre las mujeres con el ecosistema que las sustenta y da sentido a su modo de vida, a partir de los cuales su experiencia y agencia es narrada.

\subsection{Grupo de economía solidaria "Bio Saúde-Xarope e infusão de chas"}

Estudiar las mujeres rurales del sudeste de Pará nos llevó al encuentro con el grupo "Bio Saúde-Xarope e infusão de chas" en el municipio Curionópolis. Durante meses hicimos acompañamiento a sus actividades, lo que implico el contacto con otras mujeres: indígenas, quilombolas y campesinas vinculadas a la red por ellas creada para el intercambio de saberes sobre plantas medicinales.

El conocimiento de las mujeres campesinas es construido en lo cotidiano, trasmitidos de generación en generación, apoyados en la diversificación y en su relación con la biodiversidad, un aprender haciendo, relacionado con las necesidades de cada día, que respeta los tiempos y espacios de la producción y la reproducción de la vida. Es así, que interrogamos sobre las prácticas de gestión, sobre cómo se organizan, cómo surgen las ideas de hacer juntas, cuáles son las amenazas y desafíos que hoy enfrentan, sobre los significados que dan a lo que hacen, cómo ellas comprenden su propia agencia, sobre la articulación en red, la polisemia y orden práctico de la adquisición de saberes que dialogan entre sí. 
La composición del grupo es de diez personas, ocho mujeres y dos hombres, seis de ellas son monjas de la congregación "Filhas do amor divino". $\mathrm{Su}$ membresía expresa la movilidad sócio-espacial que en un pasado reciente hubo hacia ese territorio, llegadas de Río Grande do Sul, y del nordeste, su composición es heterogénea por grupos atareos y color de la piel, concentrándose el grupo superior a 50 años. Los niveles educativos son diversos, las monjas tienen nível superior y varios cursos en medicina tradicional. Cuentan con infrestructura para consultas, tratamiento y conservación de las plantas, laboratórios, pequeños huertos de plantas medicinales, espacio administrativo, entre otros.

Se consideran un grupo que funciona bajo los principios de la Economía Solidaria. Su historia no ha sido lineal, expresa un proceso de confrontación con otros poderes y resistencia dentro y fuera de la iglesia. Fuera de la iglesia, el poder municipal no valoriza el papel jugado por el grupo en el territorio, expresado en el desinterés por propuestas que articulen acciones pro salud y educación ambiental, además de las presiones que reciben de las farmaceuticas y por quienes ejercen la medicina alópata de carácter privado. Dentro de la iglesia, el giro de esta hacia una posición carismática, concentrada más en la fe que en su misión social, dejó de reconocer el accionar del grupo como parte de su trabajo pastoral. Es entonces, que bajo la orientación de la Congregación "Filhas do Amor Divino" se inscriben en SEBRAE ${ }^{11}$ como emprendimiento solidario con el nombre "Bio Saúde-Xarope e infusión de chas".

Los antecedentes del grupo se remontan a la realidad vivida en el territorio en la época del garim- po en Serra Pelada, década de los ochenta e inicios de los noventa. En condiciones de exclusión social, marcada por la pobreza, la violencia el deterioro de la salud, y la carencia de espacios educativos, fue que comenzó a gestarse en el espacio que hoy se conoce como Curionópolis la organización solidaria. Partió de la reunión de las mujeres para discutir sobre su condición

comenzamos a trabajar la cuestión de la higiene, la higiene es la puerta abierta para la salud, [...] Una gran novedad es que las mujeres no conocen su cuerpo. La menina comienza a mestruar y no sabe ni por qué mestrua y ahí comienzan las leyendas antigas. Si voce está mestruando no pode comer esto o aquello. Lo asume como enfermedad. Comenzamos a trabajar la cuestión cultural, la cuestión psicológica. La cuestión de la violencia sexual. Comenzamos un proceso de desmitificación de las enfermedades y de la concepción del corpo y de la alimentación, Entonces comenzamos a trabajar el poder de las frutas y las verduras. El valor de alimentos para la buena salud, del agua y la necesidad de ser filtrada. [...] Eso ayudó mucho. Hacíamos almuerzos comunitarios, enseñando también el aprovechamiento de las plantas para el tratamiento de las enfermedades. Cómo aprovechar la fruta toda (Entrevistada 3, 47 años, Cuirionópolis, 12/2016).

En torno al grupo se fue conformando una red de intercambio de conocimientos entre mujeres que tratan la salud con plantas medicinales en el territorio. Contaron siempre con la parcería de la Comisión Pastoral de la Tierra. Los conocimientos sobre bioenergética se articularon con los conocimientos populares que tenían en cuenta la riqueza arbolaria de la amazonia, con la dificultad de la diversidad

${ }^{11}$ SEBRAE: Servicio Brasilero de Apoyo a Micro e Pequeñas Empresas, entidad privada sen fines lucrativos. Es un agente de capacitación y promoción del desarrollo. Desde 1972, trabaja para estimular empreendedurismo y posibilitar la competitividad y sustentabilidad de pequenos grupos 
de nombres con que se pueden conocer las plantas en cada uno de los estados.

reuniamos a las mujeres para discutir su situación, sus realidades. Su reclamación eran las enfermedades que afectaban a ella y a sus familias como la malaria, dengue y anemia por la falta de alimentos. Ya conocíamos el método de la bioenergética. [...] Con la riqueza de plantas de la amazonia, enseñábamos cómo tratar la salud a través de las plantas. Teníamos dificultades para que comprendieran. Explicabamos la planta y la persona comprendía otra. Eran personas llegadas de varias regiones cada una conocia en su tierra con otro nombre y eso era una dificultad. Buscamos ayuda de biólogos en las universidades [...] vinieron especialistas para hacer corresponder el nombre cientifico con los nombres populares. Formamos una red de varios municipios que comenzó a reunirse con frecuencia para estudiar las plantas por su nombre científico. Cada mujer traía las plantas de su quintal y catalogábamos las plantas con su nombre científico y popular, ahi sabíamos qué conocimiento teníamos sobre esa planta, si era curativa o preventiva. Aprendimos mucho con el grupo de especialistas y también con el que tenían las mujeres (Entrevistada 3, 47 años, Cuirionópolis, 12/2016).

La red es heterogénea, conformada en la actualidad por hombres y mujeres de diferentes municipios del sudeste del Pará, Hay personas jóvenes y otras muy adultas, algunas representan a pueblos quilombolas o a aldeas indígenas. Sus niveles educacionales son diversos, incluyen universitarias y personas sin escolarización, en especial mujeres indígenas adultas.

La metodología seguida en cada encuentro se apoya en los presupuestos de la Educación Popular, son saberes que dialogan en condiciones de igualdad con otros saberes. Cada grupo cuenta con un tiempo para mostrar sus experiencias en el trabajo con las plantas, para la descripción de las cuáles son las dificultades de cada contexto, sus avances y cuáles son los desafíos a enfrentar. En el marco de las sesiones se discuten las necesidades de aprendizaje de cada grupo. Se cuenta con tiempos teóricos y tiempos prácticos, tales como el tratamiento a las plantas, la preparación de los jarabes y su conservación. Hay exposición, trabajo en microgrupos y devoluciones en plenaria, así como evaluación colectiva en cada cierre.

la solidaridad [...] es lo que nos hace existir, es el espíritu del cuidado a la vida humana, del cuidado a la naturaleza [...] del habitad, crecemos en conciencia y tenemos el gran desafio, quienes practicamos medicina alternativa sentimos la agresión de quienes lucran con el dolor humano [...] la medicina alópata tira de la gente no sólo dinero, tira vidas (Entrevistado 9, 58 años, Marabá, 12/2016).

Entre los aspectos más debatidos están los medioambientales, el fomento de huertos libres de agrotóxicos con plantas medicinales que cuiden de la diversidad, los desafíos que enfrentan como resistencia al modelo hegemónico y al tipo de medicina que convierte la salud en mercancía. La participación en estos encuentros nos permitió comprender el valor añadido de la palabra troca. No solo se trocan conocimientos y experiencias, se trocan sentidos, informaciones, afectos, Ello implica diálogo y negociación, se trocan desde plantas u otros productos agroecológicos hasta los propios conocimientos. 
Uno de los desafíos más serios que enfrenta el grupo es la colecta de las plantas, debido a la creciente desforestación existente en todo el sudeste del Pará $^{12}$, el avance de la megaminería, la pecuária y la soya destruyen el bioma. Los quintales y lotes tienen cada vez menos plantas, a pesar de la preocupación por su conservación. Eso implica que cada vez las plantas se localizan más lejos y a un costo mayor.

El tiempo también es un desafío. En un momento en que aumenta las demandas de tratamiento por las enfermedades causadas en lo fundamental por la explotación mineral, por prácticas tradicionales agrícolas como la quema en determinada época del año o por el uso de químicos en los cultivos, $u$ otras convertidas en epidemias como el dengue o el chikungunya, que demandan tiempo de atención, resta poco para otras actividades como la colecta de plantas, el registro y sistematización de la experiencia o participación en congresos.

tenemos que colectar plantas, hacer la contabilidad, administrar y todas las hermanas tienen más de 55 años, eso las limita en salir a realizar otros trabajos. No conseguimos personas que administren como nosotros, no se puede pensar solo en lo financeiro, tenemos una ideología que no es el dinero. La importancia de curar la persona que viene con la salud fragilizada es nuestro objetivo, pero tenemos que mostrar por nuestro testimonio que acreditamos en una forma diferente de vivir y eso toma mucho tiempo [...]. Estamos tentando encontrar personas que no vea en el dinero lo principal, que sueñen lo mismo que la gente sueña, no somos una empresa de vender remedio, no pensamos en el lucro. El conoci- miento es una troca. La gente va viendo el presente y no conseguimos tiempo (Entrevistada 3, 47 años, Cuirionópolis, 12/2016).

Si analizamos la actividad realizada por el grupo a la luz de las teorias del cuidado, en un contexto tan patriarcal como son los espacios mineros y de agronegócio, podemos encontrar siguiendo a Carol Gilligan (2013) una ética del cuidado como consagración al projimo en manos de estas mujeres, una ética asumida con responsabilidad e interdependencia. Sin pretender ver aqui una naturalización de la experiencia de cuidado, sino la fragilidad de su dimensión institucional y pública, y por tanto democrática. El cuidado así ejercido combina sentimentos de afecto y responsabilidad por los otros. La red incluye toda una ética de ecodependencia y de co-responsabilidad, que se extiende a quien recibe los benefícios del cuidado. Hay una respuesta a su recibimiento; podríamos encontrar así a nível muy micro determinados ejemplos de democratización del cuidado o de la asistencia democrática ya referidas por Tronto (2007).

A nivel de las relaciones establecidas se fomenta una cadena productiva en las redes de cuidado, basada en la solidaridad. La consulta y los medicamentos preparados contienen más valor de uso que valor de cambio. Cuando las personas no pueden costear monetariamente, ellos traen plantas en condición de una troca.

\footnotetext{
${ }^{12} \mathrm{El}$ "Instituto do Homen e Meio Ambente da Amazonia" (IMAZON) en sus publicaciones de sobre el desmatamento de la Amazonia Legal viene apuntando al Pará como uno de los estados responsable por la desforestación, debido a la expansión de la minería, la pecuaria extensiva y más recientemente la introducción del cultivo de la soya. De los $2441 \mathrm{~km} 2$ de área desforestada entre agosto de 2017 y mayo 2018,852 fueron en Pará, correspondendo el 52\% a áreas protegidas. Ver boletim do desmatamento da Amazônia Legal (IMAZON, 2018).

https://imazon.org.br/publicacoes/boletim-do-desmatamento-da-amazonia-legal-agosto-2018-sad/
} 
[...] cuando se sienten curados, ellos continúan donando las plantas, cuando vienen pequeños propietarios también se colocan a disposición de donar en función de la salud de los demás, o lo hacen a precios justos. Cobramos lo mínimo por el atendimiento. La solidaridad está en la base. Cuando las personas no tienen dinero se troca por plantas. Conseguimos miel agroecológica en los asentamientos, o compramos productos en otros emprendimientos como a la cooperativa de las Quebradeiras de Coco Babaçu que radica en San Domingo de Uruguaya (Entrevistada 3, 47 años, Cuirionópolis, 12/2016).

Se trata de un intercambio que emerge de prácticas tradicionales, realizada entre organizaciones no-capitalistas, apoyadas en la solidaridad, una otra forma de hacer economia, cimentada en el bienestar humano.

\subsection{Cooperativa "Sabores da Serra" en la herida abierta en la Selva Amazónica ${ }^{13}$ conocido por "Serra Pelada"}

Su ubicación es en el município Curionópolis a $80 \mathrm{~km}$ de la ciudad de Marabá en el sudeste del Pará, un espacio en que confluyen intereses entre la Empresa Vale S A, el gobierno federal y más de cien mil garimperos organizados en cooperativas. Es aquí donde un grupo de mujeres de diferentes generaciones, negras en más del 70\%, llegada casi todas de la región nordestina en la época de funcionamento del garimpo busca en la solidaridad formas de agencia para la supervivencia de sus famílias. Comenzaron por pasar cursos sobre el trabajo con la fibra de banana y para el aprovechamiento de la fruta, así se fueron definiendo como un grupo productor de dulces, utilizando la banana y otras frutas.

$\mathrm{Su}$ trayectoria fue de avances y de retrocesos, de salidas y de entradas al grupo, de frustraciones y de pequeños éxitos, primero eran 54 mujeres, su número fue disminuyendo hasta quedar en 25 . Experiencia que viene del apoyo mutuo en las salidas colectivas en busca de pequenos gramos de oro que les permitiera algún ingreso. Si en los momentos del garimpo esa era una táctica de proteción frente a la violencia patriarcal, ahora juntas arrancan migajas a la Empresa Vale S. A. como es la conquista de un espacio de tierra para la siembra de banana y también de un determinado financiamiento para el montaje de la fábrica de dulces. Si bien, puede entenderse el fondo otorgado como responsabilidad social de la mineradora o como proceso de cooptación, lo certo, es que las mujeres diseñan estratégias para generar ingresos. Es una negociación colectiva de resistencia, si tenemos en cuenta, que sus trayectorias pasadas las vinculan al garimpo, la mayoria de ellas viudas reclaman una compensación financeira, lo que las lleva a participar de otras organizaciones colectivas, como las asociaciones de vecinos, y las mismas cooperativas de garimperos.

La cooperativa fue fundada en 2005 y conjuga saberes tradicionales con otros adquiridos.

buscabamos una salida a nuestra situación, conseguimos primero el espacio para la siembra de bananas, luego vinieron los equipos para riego, y empezamos a buscar conocimientos para trabajar la fibra y el procesamiento de la fruta con apoyo de organizaciones de la sociedad civil (Entrevistada 10, 57 años, Curionópolis, 02/2017).

${ }^{13}$ Así denominó Ricardo Kotscho (1984) el espacio de garimpo: Serra Pelada: uma ferida aberta na selva. 
Si bien, la organización les permitió generar determinada autonomia, la significación atribuida va mucho más allá. "el sufrimiento queda en casa, olvidamos. Aqui la gente sonrie, aprendi mucha cosa con estas mujeres y el barco va para frente" (Entrevistada 11, 55 años, Serra Pelada, 11/2016). "Quien trabaja sabe el valor del cooperativismo, [...] lo que estoy valorizando es el conocimiento, estamos aprendiendo a andar con nuestras propias rendas, con momentos tristes y momentos buenos" (Entrevistada 12, 48 años, Serra Pelada, 11/2016).

El emprendimiento no es sólo un espacio de producción, es un punto de encuentro, de reunión, de co-aprendizanjes, de escucha, donde crece la autoestima y se gana autonomia, cuestión relevante para quienes responden por la reproducción de la vida.

En las asambleas conversamos de nuestros sufrimientos en casa, de la droga, la prostitución o de nuestras hijas adolelescentes ambarazadas, de las violencias sufridas como mujeres, de cómo seguir para frente, me gusta escuchar, quedo nerviosa, creamos pequeños grupos para aconsejar y luego ver quien habla por el grupo (Entrevistada 13, 53 años, Serra Pelada, 02/2017)

Ellas, madres de entre cuatro y seis hijxs, responden por las atividades de cuidado dentro y fuera de los hogares. Ello aún es más difícil cuando se trata de cuidar a personas con enfermedades negligenciadas.

Enfermamos debido al fracaso de los sueños, no tenemos salida, esperamos la indennización que no llega. Vivimos sobre una tierra rica en hierro y oro, pero somos muy pobres. Ellos explosionan y contaminan el agua, la tierra, el aire.Vivimos en un ambiente contaminado por mercurio. Cuando colocan dinamita todo estremece, somos rehenes de esa empresa. Ahora nuestro sueño es que la cooperativa vaya para frente (Entrevistada 14, 61 años, Serra Pelada, 02/2017)

Las narrativas construídas muestran los desafios en que se debaten estas mujeres, los riesgos del dia a dia por los impactos de la minería y la provisionalidad de su existencia "nadie vino por el resto de la vida, nadie construyó casas, sino barracos de madera, todos pensaban en irse, mi madre vino por seis meses y hasta hoy no tiro eso de la cabeza. Desde 1992 esperamos por indennización. Precisamos ver el final de esta historia" (Entrevistada 15, 55 años, Serra Pelada, 10/2016).

Para responder entonces, a la pregunta ¿por qué los conflictos socioambientales generados por la minería son también una cuestión feminista? nos remitimos al substrato teórico legado por el ecofeminismo crítico y tercermundista para hacer una lectura de las posturas que nacen del protagonismo de las mujeres en la defensa de su territorio y de sus cuerpos-territorios ${ }^{14}$, con un lenguaje de valoración anclado en las problemáticas del contexto, relacionado con la sobrevivencia, el cuidado de si y de la naturaleza.

Cuando en de las narrativas se expresa que su lucha es porque, "queremos a las mujeres y a las palmeras vivas" está contenida aquí la resistencia a la violencia patriarcal que los conflictos socioambientales inscriben en sus cuerpos. Una lógica de dominación que relaciona los procesos

\footnotetext{
${ }^{14}$ Tesis desarrollada Lorena Cabnal (2015), feminista comunitaria guatemalteca quien valora que somos cuerpos arraigados a territorios; por lo tanto, lo que pase en los cuerpos afecta a los territorios y viceversa.
} 
desposesión del territorio con la dominación sobre las mujeres, justificando así, las jerarquías legadas por el pensamiento moderno occidental sobre las mujeres y la naturaleza.

Tradicionalmente las mujeres amazónicas construyeron sus medios de vida a través de un entretejer los ecosistemas con las relaciones sociales construidas entre las mujeres. La violencia estructural agudizada por la minería conduce a la pérdida del territorio y por tanto anula el aprovechamiento de sus bienes forestales; plantas medicinales, fuentes de agua, ect.; Cuando expresan que tienen más dificultad en conseguir las plantas, o que el acceso al coco babaçu es cada vez más distante, o de la pérdida de árboles tradicionales de los que extraen el aceite como andiroba o castaña, o nos hablan de las enfermedades que aparecen, o las presiones y despojos en sus territorios, nos están indicando cuan desventajosa es la minería para la vida de las mujeres, acentuando las desigualdades de género y por tanto su condición subordinada.

Las narrativas y fuentes consultadas muestran las conexiones existentes entre el avance del (neo) extractivismo, las tensiones socioambientales y las desigualdades de género; como también muestran el protagonismo de las mujeres en las resistencias.

Ellas salen del ámbito doméstico, crean sus propias organizaciones, redes de solidaridad y apoyo, se movilizan, obtienen sus propios recursos, denuncian la violencia de género que las afecta, logran visibilidad, cuestionan al poder local, al tiempo que se posicionan políticamente ante la necesidad de preservar sus territorios y el ecosistema del que depende la reproducción de sus vidas. Lo que nos lleva a definir su praxis como ecofeminista, una praxis que reivindica derechos al uso del territorio, al disfrute de su mundo simbólico y a su existencia como mujeres.

\section{Conclusiones}

Comprender la experiencia y la agencia de mujeres rurales en espacios mineros en el sudeste del Pará significó seguir la ruta de sus trayectorias, cuyas narrativas se movían de manera artesanal entre lo individual y lo colectivo, entrecruzadas con el contexto situacional. A través del contenido de lo narrado y lo vivido apreciamos las formas en que expresan su agencia, cuya máxima expresión son las organizaciones colectivas en condiciones de extrema vulnerabilidad impuestas por las lógicas en que ellas y el capital se relacionan con el territorio y la naturaleza. Si para ellas la naturaleza es modo y fuente de vida, para el capital se torna espacio objeto, apropriado y mercantilizado, lo que muestra el desequilibrio en las relaciones de poder.

Sus experiencias están relacionadas a la lucha por la reproducción y el cuidado de la vida, es una lucha por el derecho a ser y existir como mujeres. Las prácticas socioproductivas, desarrolladas las vinculan al uso de los bienes que brinda el bioma amazónico y por tanto, están interesadas en su gestión sostenible. Las mujeres tienen un conocimiento que es diferenciado, relacionado con las maneras con que sus prácticas cotidianas las ha vinculado al cuidado y sostenibilidad de la vida.

Espacios de accionar colectivo como los estudiados, más allá de los objetivos de generación de renta, son espacios de participación y gestión democrática, de solidaridad, autonomía y de problematización de las condiciones y posicionalidades como mujeres. La diversidad de trayectorias de vida 
marcadas por la migración y la pobreza, no fue un obstáculo para articularse en torno a acciones colectivas de resistencia ante el control que ejerce el modelo hegemónico capitalista y patriarcal sobre la tierra y los bienes que brindan los bosques amazónicos

El espacio colectivo de las mujeres se convierte así, en un territorio conquistado, un espacio de producción y de sueños en que las mujeres re-significan sus propias existencias. Los cursos recibidos y las decisiones tomadas se constituyen en herramientas para problematizar el contexto.

Sus narrativas permiten comprender la experiencia y agencia de mujeres cuya estrategia colectiva expresa resistencia a la rapacidad con que el modelo dominante mercantiliza la naturaleza y la salud humana, asumiendo que lo natural también es político. Las maneras de gestionar, producir, de compartir e intercambiar aspectos materiales y espirituales constituyen chispazos de solidaridad, una flor nacida en el estercolero neoliberal. Sus prácticas están vinculadas al cuidado, a la reproducción social de la vida y a una economía cuya importancia quedó invisibilizada en la reproducción del capital.

Los hechos muestran que el avance de la explotación minera en el contexto estudiado genera violencia y lucro, en la misma medida que desvaloriza aquellos sistemas productivos tradicionales apoyados en el bioma amazónico, que reproducen la vida y generan renta, desempeñadas en la mayoría de los casos por las mujeres. Las resistencias al neoextractivismo desde la experiencia y la agencia de las mujeres hace que emerjan posturas feministas cuestionadoras del sistema de dominación sexo-género; muchas veces sin autodefinirse como tales; posturas que redimensionan el cuidado, la salud comunitaria, la supervivencia, así como la defensa del territorio y de una otra ontología que revaloriza las relaciones las relaciones con la naturaleza en un sentido de ecodependencia.

Excluidas de los llamados "benefícios" del modelo desarrollo impuesto por la modernidad occidental, ellas reinventan sus trayectorias en la misma medida que diseñan estrategias colectivas que resisten a los procesos de apropiación y desterritorialización que el (neo)extractivismo trae consigo.

\section{Referencias}

Brah, A. Cartografias de la diáspora: Identidades en cuestión. Madrid: Traficantes de Sueños, 2011.

Brasil. Câmara dos deputados. Comissão aprova Projeto Lei do Babaçu Livre, Projeto de Lei 231/2007. Disponible en: <http://www2.camara.leg.br/atividade-legislativa/ comissoes/comissoes-permanentes/cmads/documentos/ 2007-08-09-comissao-aprova-lei-do-babacu-livre>. Consultado en: set. 2015.

Brito, D. C. A paradoxal unidade do discurso do desenvolvimento. In: Altvater, E. Terra incógnita: reflexões sobre globalização. Belém: UFPA-NAEA, 1999. p. 175-202.

Brito, M. F de. Mulheres e mineração no Brasil. Rio de Janeiro: IBASE, 2016.

Cabnal, L. Corps-territoire et territoire-terre: le féminisme communautaire au guatemala. Entretien avec Lorena Cabnal, 2015. Disponible en: <https://www.cairn.inforevuecahiers-du-genre-2015-2-page-73.htm $\geq$. Consultado en : set. 2018.

Coelho, T. P. Projeto Grande Carajás: trinta anos de desenvolvimento frustrado. In: Zonta, M.; Trocate, C. H. (Orgs.). A questão mineral no Brasil. Marabá, Pará: Editorial Marabá, v. 1, 2015. p. 27-158.

CPT - Comissão Pastoral da Terra. Conflitos no campo 2016. Goiânia-GO: Centro de Documentação Dom Tomás Balduino, 2017. Disponible en: <www.cptnacional.org. br/publicacoes-2/destaque/3727-conflitos-no-campo-bra- 
sil-2016>. Consultado en: mai. 2018.

Congilio, C. R; Bezerra, R.; Michelotti, F. (Org.). Mineração, trabalho e conflitos amazônicos no sudeste do Pará. Marabá, PA: Iguana, 2019. Disponible en: https://br.boell. org/sites/default/files/2020-04/1562059330971_MinerTrabConflitos_ebook-1.pdf $\geq$. Consultado en: dez. 2019.

Congilio, C. R.; Moreira, E. S. La Resistencia A Los Grandes Proyectos De Explotación De Hierro En El Sudeste Paraense. In: Feliz, M.; Torno, C. (Orgs.). El neodesarrollo en debate: crisis, transición y alternativas. Buenos Aires-Argentina: El Colectivo, v. 1, 2017, p. 77-92.

Crenshaw, K. W. Mapping t.e margings. Intercetionality, Identtity Politics, and Violence agains Women of Color. Stanford Law Review, 43(6), p. 1.241-1.299, 1991. Disponível em: http://www.jstor.org/stable/1229039?origin=JSTOR-pdf. Consultado en: mai. 2017.

Curvina, E. J.; Moreira, E. S. Os Deslocamentos Provocados pela Hidrelétrica de Tucuruí no Pará: Reflexões Sobre Jacundá E Arraias. Agenda Social, 12(1), p. 52-67, 2018.

De la Cuadra, F. Pueblos originarios, conflicto socio-ambiental y postdesarrollo en America Latina. Ambiente \& Sociedade, XVIII(2), 23-42, 2015. doi: 10.1590/1809-4422ASOCEx02V1822015en

Escobar, A. Una minga para el postdesarrollo: lugar, medio ambiente y movimientos sociales en las transformaciones globales. Lima: Universidad Nacional de San Marcos, 2010.

Esteva, G. Más allá del desarrollo: la buena vida. Revista América Latina en Movimiento, 445, 1-5, 2009. Disponible en: https://www.alainet.org/sites/default/files/alai445w.pdf

Gilligan, C. La ética del cuidado. Barcelona: Cuadernos de la Fundación Víctor Grífols, 2013.

Gudynas, E. Diez tesis urgentes sobre el nuevo extractivismo: contextos y demandas bajo el progresismo sudamericano actual. In: Schuldt, J.; Acosta, A.; Barandiarán, A.; Folchi, M; Alayza, A.; Gudynas, E. Extractivismo, politica y sociedad. Quito: Centro Andino de Acción Popular (CAAP) y Centro Latino Americano de Ecología Social (CLAES), 2009a. p. 187-225. Disponible en: http://www.gudynas. com/publicaciones/GudynasNuevoExtractivismo10Tesis09x2.pdf Consultado en: nov. 2018.
Gudynas, E. El día después del desarrollo. Agonía de um mito ¿Cómo reformular el "desarrollo"? Revista América Latina en movimiento, 445, 31-33, 2009b. Disponible en: Disponible en: https://www.alainet.org/sites/default/files/ alai445w.pdf

Gudynas, E. Si eres tan progresista ¿Por qué destruyes la naturaleza? Neoextractivismo, izquierda y alternativas. Ecuador Debate, 79, 61-81, 2010. Disponible en: https:// www.caapecuador.org/ecuador-debate-n-79/CConsultado en: nov. 2017.

Guevara, M. Experiencias agroecológicas amazónicas protagonizadas por mujeres en el Sudeste de Pará, Brasil. In: Zuluaga, P.; Cataroga-Vargas, G.; Siliprandi, E. (Coords.). Agroecologia en femenino: reflexiones a partir de nuestras experiencias. La paz: SOCLA, 2018. p. 107-122.

Hall, A. L. Amazônia, Desenvolvimento Para Quem? Desmatamento e Conflito Social no Programa Grande Carajás. Rio de Janeiro: Jorge Zahar, 1991.

Hebette, J. Cruzando fronteira: 30 anos de estudo do campesinato na amazonia. Belém: Editora Universitária, UFPA, v. 3, 2004.

IBASE - Instituto Brasileiro de Análises Sociais e Econômicas. Contradições do desenvolvimento e o uso da CFEM em Canaã dos Carajás, 2018. Disponible en: $<$ http://ibase. $\mathrm{br} / \mathrm{pt} /$ noticias/debate-sobre-uso-da-cfem-em-canaa-dos-carajas-pa-mobiliza-atores-locais/>. Consultado en: oct. 2018.

IBGE - Instituto Brasileiro de Geografía e Estaística. Cidades estimativa da população em 2015, 2015. Disponible en: $<$ https://cidades.ibge.gov.br>. Consultado en: ago. 2017.

Ikeda, J. O. C. Expansão do capital, luta de classes e poder local na Amazônia: um Estudo Sobre a Composição do Poder Político no Município de Marabá/Pará (1985-2012). Marabá, Dissertação (Mestrado em Dinâmicas Territoriais e Sociedade na Amazônia) - Unifesspa, 2014.

IMAZON - Instituto do Homen e Meio Ambente da Amazonia. Boletim do desmatamento da Amazônia Legal, 2018. Disponible en: $<$ https://imazon.org.br/publicacoes/boletim-do-desmatamento-da-amazonia-legal-agosto-2018-sad/ $\geq$. Consultado en: oct. 2018.

Kotscho, R. Serra Pelada: Uma ferida aberta na selva. São 
Paulo: Brasiliense, 1984.

Lauretis, T. de. Alice doesn 't. Indiana: University Press, 1984.

Lugones, M. Colonialidad y Género. Tabula Rasa, 9, 73101, 2008. Disponible em: https://www.revistatabularasa. org/numero-9/05lugones.pdf

Mahmood, S. Teoría feminista, Agência e sujeto liberatório: algumas reflexôes sobre o revivalismo islâmico no Egipto. Etnográfica, 10(1), 121-158, 2006. doi: 10.4000/ etnografica.6431

Max-neef, M. Economía descalza: señales desde el mundo invisible. Montevideo: Editorial Nordam Comunidad, 1986.

Mohanty, C. T. Bajo los ojos de occidente. In: Suárez, N. L.; Hernández, A. (Eds.). Descolonizando el Feminismo: teorías y prácticas desde los márgenes. Madrid: ed. Cátedra, 2008. p. 112-161.

Peña-Vega, A. Meta-desenvolvimento, auto-organização e incerteza: um caminho em direção ao pensamento ecológico. In: Castro, E.; Pinton, F. (Orgs.). Faces do trópico úmido: conceitos e novas questões sobre desenvolvimento e meio ambiente. Belém: Cejup; UFPA-NAEA, 1997. p. 201-220.

Pérez Orozco, A. La sostenibilidad de la vida en el centro ¿y eso que significa? In: Mora, L.; Cabello de, A.; Escribano, J. (Coords.). La ecología del trabajo: el trabajo que sostiene la vida. La Rioja: Fundación Dialnet, 2015. p. 71-100.

Pinassi, M. O.; Cruz Neto, R da. A mineração e a lógica da produção destrutiva na Amazônia. In: Trocate, C.; Zanon, M.; Vieira, J. (Orgs.). Elementos constitutivos do Movimento anti Mineraçâo. Marabá: Editorial Iguana Reflexão Amazônica, 2015. p. 80-99.
Santos, A. A. S. dos. Mineração e Conflitos Fundiários no Sudeste Paraense. Marabá, Dissertação (Mestrado em Dinâmicas Territoriais e Sociedade na Amazônia) - Unifesspa, 2018.

Segato, R. Femi-geno-cidio como crimen en el fuero internacional de los Derechos Humanos: el derecho a nombrar el sufrimiento en el derecho. In: Fregoso, R.; Bejarano, C. (Eds.). Feminicidio en América Latina. México, DF: UNAM-CIIECH;Red de Investigadoras por la Vida y la Libertad de las Mujeres, 2011.

Shiva, V. El mundo en el límite. In: Giddens, A.; Hutton, W. (Eds.). En el límite: la vida en el capitalismo global. Barcelona: Tusquets, 2001.

Shiva, V.; Mies, M. Ecofeminismo: teoría, crítica y perspectivas. Barcelona: Icaria Editorial, 2013.

Silvestre, M. do P. S. A.; Lima, L. N. G. C. Hanseníase: considerações sobre o desenvolvimento e contribuição (institucional) de instrumento diagnóstico para vigilância. Revista Pan-Amazônica de Saúde, 7, 93-98, 2016. Disponible en: http://scielo.iec.gov.br/pdf/rpas/v7nesp/2176-6223-rpas-7-esp-00093.pdf

Svampa, M. Extractivismo neodesarrollista y movimientos sociales. ¿Un giro ecoterritorial hacia nuevas alternativas? In: Grupo Permanente de Trabajo sobre Alternativas al Desarrollo. Más allá del desarrollo. Quito: Ediciones Abya Yala, 2011. p. 185-218.

Tronto, J. Assistência democrática e democracias assistenciais. Sociedade e Estado, 22(2), 285-308, 2007. Disponible en: https://periodicos.unb.br/index.php/sociedade/article/ view/5334/4852

UNESCO - Organização das Nações Unidas para a Educação, a Ciência e a Cultura. Different theories and pratices of development. Nueva York: UNESCO, 1982. 